\title{
Random Vector Functional Link Network Optimized by Jaya Algorithm for Transient Stability Assessment of Power Systems
}

\author{
Jianhong Pan, Jiashu Fan, Aidi Dong, and Yang Li iD \\ State Grid Jilin Electric Power Company Limited, Changchun 130000, China \\ Correspondence should be addressed to Yang Li; 876464104@qq.com
}

Received 29 September 2020; Revised 21 November 2020; Accepted 25 November 2020; Published 7 December 2020

Academic Editor: Ying-Yi Hong

Copyright (c) 2020 Jianhong Pan et al. This is an open access article distributed under the Creative Commons Attribution License, which permits unrestricted use, distribution, and reproduction in any medium, provided the original work is properly cited.

\begin{abstract}
A novel transient stability assessment (TSA) approach using random vector functional link (RVFL) network optimized by Jaya algorithm, called Jaya-RVFL, is proposed for power systems in this paper. First, by extracting system-level features from phasor measurement unit (PMU) measurements as predictors, an RVFL-based TSA model is proposed. In order to improve the performance of RVFL classifiers, a quantile scaling approach is utilized to optimize the randomization range of input weights via the Jaya algorithm. The simulation results on IEEE 39-bus system and a real-world power system show that the presented method outperforms other popular methods comprising multilayer perception, probabilistic neural network, and support vector machine.
\end{abstract}

\section{Introduction}

Transient stability assessment (TSA) has long been considered to be of paramount importance for ensuring safe operation of power systems [1]. Transient stability refers to the ability of an electric power system to remain in synchronism between machines after suffering from serious disturbances $[2,3]$. Problems arising from the growing integration of intermittent renewable power generation in a variety of forms, such as active distribution networks [4], microgrids [5], and integrated energy systems [6], are pushing a power system to complex and variable operating scenarios due to the inherent uncertainties of renewable generation [7]. Meanwhile, the increasing cyberattacks and the reliance on control and communication are resulting in new sources and propagation paths of cascading failures [8]. As a new generation of DC transmission technique, voltage source converter-based highvoltage direct current (VSC-HVDC) has become a popular opinion of power transmission due to its significant advantages such as independent adjustments of active and reactive powers [9] and asynchronous interconnection between islands [10]. Besides, the integration of energy storage [11, 12], electric vehicles [13], and increasingly diversified demands $[14,15]$ affect the stable operation of the system to a certain extent. All these shifts pose new challenges in maintaining the system working reliably [16]. Recently, the successful application of time-stamped phasor measurement units- (PMUs-) based wide-area measurement system (WAMS) makes synchronized measurements available for use, which opens up new opportunities for developing a modern wide-area protection and control (WAPaC) system [17-19].

In general, the TSA methods consist of two categories: time-domain (T-D) simulations [20] and direct method [21-24]. Machine learning has proven as an effective way to solve complex electrical engineering problems $[25,26]$. Recent research demonstrates that pattern recognition techniques, such as decision trees (DT), artificial neural networks (ANN), and support vector machines (SVM), are promising for assessing transient stability status of power systems, called pattern recognition-based TSA (PRTSA) [27]. (1) DT-based TSA: In [28], a dynamic security assessment approach is proposed by using PMUs and DT; and then, an adaptive ensemble DT is developed for doing so in [29]; in [30], a generic DT-based probabilistic framework is put forward for predicting transient stability of power systems via PMU data. (2) ANN-based TSA: ANN is originally introduced to solve TSA problems by Sobajie and Pao in [31]; in [32], an ANNbased dynamic security assessment method is proposed for power systems with fisher discrimination-based feature selection; in [33], a TSA approach based on recurrent artificial 
neural network is presented for multimachine power systems; in [34], multilayered perceptron (MLP) neural networks are utilized for TSA of power systems with detailed models; and in [35], a TSA approach is developed by using a hybrid intelligent system consisting of a preprocessor, an ANN array, and an interpreter. (3) SVM-based TSA: in [36], an SVMbased TSA approach is put forward for large-scale power systems; in [37], an SVM-based algorithm is proposed for TSA via PMU data; in [38], a feature selection approach based on improved maximal relevance and minimal redundancy (mRMR) coupled with SVM-based classifier is proposed for TSA; and in [39], energy-based features are employed as the inputs of an SVM classifier for TSA. (4) Extreme learning machine- (ELM-) based TSA: in [40], a real-time TSA model using ELM is presented; in [41], an ELM-based TSA model optimized by improved particle swarm optimization (IPSO) is proposed by using PMU data; in order to address the issue of lacking online learning ability in current PRTSA methods, an ELM-based critical clearing time forecasting method is presented for power systems in [42]; in [43], an intelligent system via an ensemble of OS-ELM (EOS-ELM) is developed for TSA; in [44], a real-time EOS-ELM-based TSA method with Jaya-based feature selection is put forward; in [45], a rule extraction method is developed by using ELM and improved ant-miner algorithm for TSA. (5) Other PRTSA approaches: in [46], a core vector machine-based TSA is presented. In [47], a multifeature fusion approach for TSA is proposed via PMU data. In [48], a time-adaptive TSA approach is developed. Unfortunately, there are some inherent disadvantages in PRTSA methods. For example, the ANN has problems of overfitting, local optima, and slow convergence; the SVM has difficulty in optimal parameter selection. These abovementioned defects seriously limit the practical application of such methods.

Random vector functional link (RVFL) network originally proposed in [49] is a powerful single-layer feedforward neural network (SLFN) with significant advantages of efficient training speed and universal approximation [50], and it has been widely used for addressing various power system issues such as voltage stability assessment [51], static security assessment [52], and dynamic security assessment [53]. Meanwhile, the Jaya algorithm proposed in [54] is an emerging intelligent optimization algorithm, whose optimization performances do not rely on the algorithm-specific control parameters [55]. The Jaya algorithm has been employed to solve complex optimization problems in engineering, such as optimal parameter selection [44], optimal power flow [56], and optimization of thermal devices and cycles [57]. In this paper, a novel TSA approach using RVFL network optimized by using the Jaya algorithm, called Jaya-RVFL, is proposed for power systems. First, by extracting system-level features from PMU measurements as predictors, an RVFL-based TSA model is proposed. For the purpose of improving the performance of RVFL classifiers, a quantile scaling approach is utilized to optimize the randomization range of input weights via the Jaya algorithm.

The rest is structured as follows: the construction of the original feature set is presented in Section 2, and then the principle of the proposed Jaya-RVFL-based TSA approach is presented in detail in Section 3. Application of the approach is shown using the IEEE 39-bus system and a practical system in Section 4. At last, Section 5 draws the conclusions.

\section{Construction of the Original Feature Set}

It is an important task for PRTSA to construct effective original features $[58,59]$. Due to limitations of traditional measurement systems, previous works tend to utilize prefault static features as predictors; while this work focuses on extracting system-level features from postfault dynamic information from WAMS.

2.1. WAMS. Due to the limitations of traditional measurement systems like SCADA, existing works tend to utilize prefault static features. The industrial application of WAMS has recently broken through this bottleneck, whose structure is illustrated in Figure 1.

As shown in Figure 1, WAMS is a measurement system for a power system including multiple areas, and it typically consists of three components: PMU, communication system, and control system. Just because WAMS is capable of supplying postfault synchronized measurements, this work focuses on extracting features from PMU data as predictors for real-time TSA.

2.2. Original Feature Set. In order to comprehensively depict the transient disturbed pattern space during the whole distribution process, the original features are extracted from different phases including the prefault steady state, fault clearing time, and postfault state. As listed in Table 1, the input features proposed in $[58,59]$ are used as the original input features of this work.

\section{Principle of the Proposed Approach}

3.1. RVFL Network. As illustrated in Figure 2, RVFL network has three layers: input, hidden, and output layers. Different from other SLFNs like ELM, there are direct links between the input and hidden layers in the RVFL. For a given training set $D$ with $N$ instances in the following form: $D=\left\{\left(\mathbf{x}_{i}, \mathbf{y}_{i}\right) \mid \mathbf{x}_{i} \in \mathbf{R}^{M}, \mathbf{y}_{i} \in \mathbf{R}^{P}\right\}, \quad i=1, \cdots, N, \quad x_{i}=\left[x_{1}, \cdots\right.$, $\left.x_{m}, \cdots, x_{M}\right] \in \mathbf{R}^{M}$ and $\mathbf{y}_{i}=\left[y_{1}, \cdots, y_{p}, \cdots, y_{P}\right] \in \mathbf{R}^{P}$ are the $i$ th input vector with $M$ input features and the $i$ th output vector with $P$ output features. The RVFL network with $L$ hidden nodes is mathematically written as follows: where $h_{l}=f\left(\sum_{m=1}^{M} \alpha_{m, l} x_{m}+b_{i, l}\right) ; \alpha_{m, l}$ and $\beta_{l, p}$ are, respectively, the weights between input and hidden layers and the weights between hidden and output layers; $\beta_{m, p}$ are the weights between input and output layers; $f$ is the activation function; and $b_{i, l}, b_{i, p}$, and $b_{h, p}$ are the biases between layers.

$$
y_{p}=\sum_{l=1}^{L} \beta_{l, p} h_{l}+b_{h, p}+\sum_{m=1}^{M} \beta_{m, p} x_{m}+b_{i, p}
$$




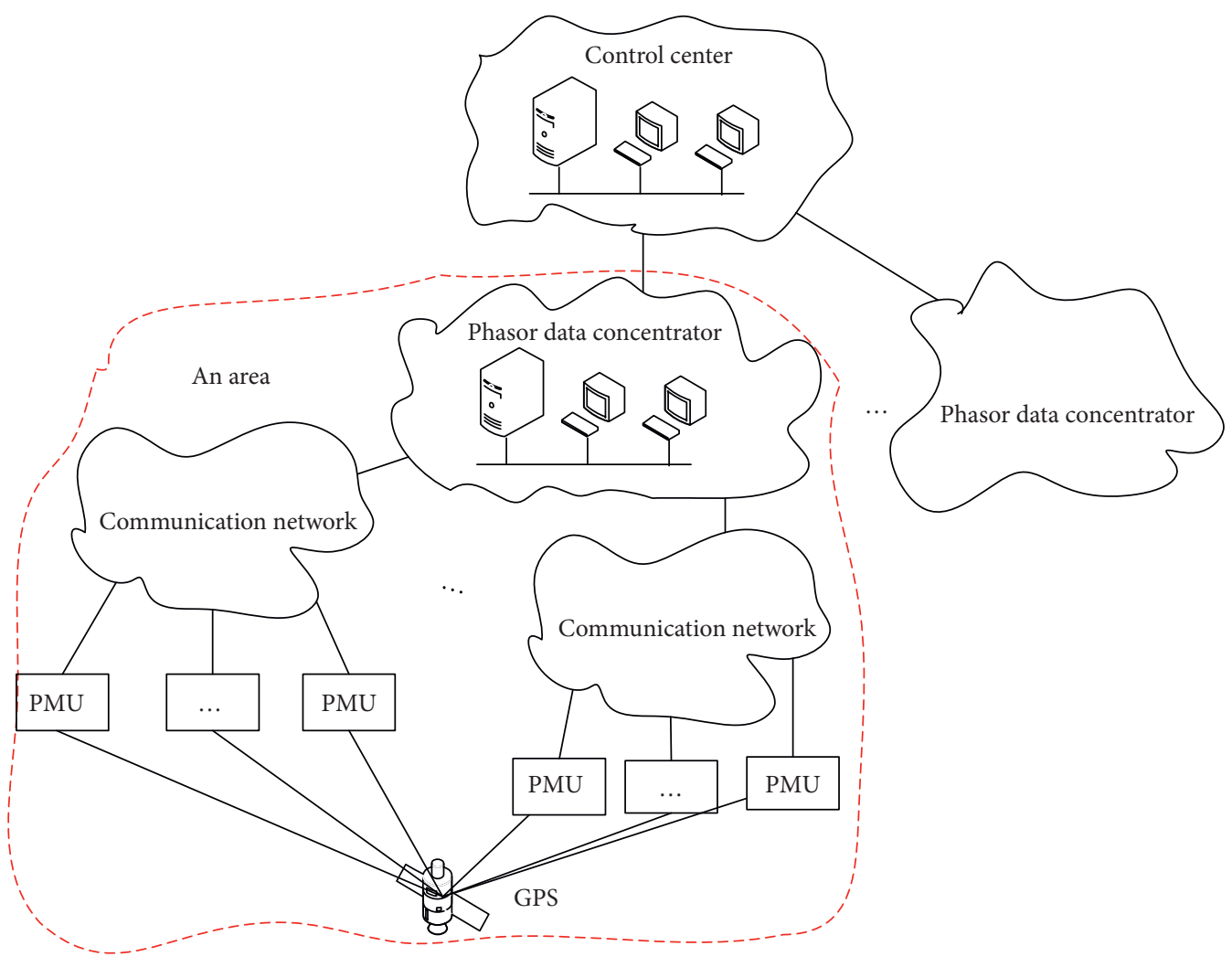

FIgURE 1: Structural diagram of WAMS.

Recent research in [50] suggests that the output bias terms are insignificant; therefore, they are omitted, i.e., $b_{i, p}=b_{h, p}=0$. For ease of description, (1) is rewritten as

$$
\mathrm{H} \boldsymbol{\beta}=\mathrm{Y},
$$

where $\mathbf{H}$ is the hidden layer output matrix, $\mathbf{Y}=\left[\mathbf{y}_{1}, \cdots, \mathbf{y}_{N}\right]^{T}$, and $\beta$ is the output weight vector.

And finally, one can obtain

$$
\widehat{\boldsymbol{\beta}}=\mathbf{H}^{\dagger} \mathbf{Y},
$$

where $\mathbf{H}^{\dagger}$ is the Moore-Penrose generalized inverse of $\mathbf{H}$.

3.2. Jaya Algorithm. The key idea of this algorithm is that a solution should move to the optimal solution and away from the worst one [54], which is represented as

$$
X_{j, k, i}^{\prime}=X_{j, k, i}+r_{1, j, i}\left(X_{j, \text { best }, i}-\left|X_{j, k, i}\right|\right) \times r_{2, j, i}\left(X_{j, \text { worst }, i}-\left|X_{j, k, i}\right|\right),
$$

where $X_{j, k, i}$ denotes the value of variable $j$ for candidate $k$ at iteration $i ; X_{j, k, i}^{\prime}$ refers to the updated value of $X_{j, k, i}$; $X_{j, \text { worst }, i}$ and $X_{j, \text { best }, i}$ are, respectively, the values of variable $j$ for the worst and best candidates [44]; and $r_{1, j, i}$ and $r_{2, j, i}$ are the two random numbers for variable $j$ at iteration $i . X_{j, k, i}$ is accepted, if this updated value yields a better function value. More details of this algorithm can be found in $[44,54]$.

\subsection{Jaya-RVFL-Based TSA}

3.3.1. Jaya-Based RVFL Training. According to $[50,53]$, the quantile scaling algorithm is an effective way to improve the performance of RVFL networks by optimizing the randomization range of input weights. In this study, the Jaya algorithm is employed to optimize the two parameters: the regularisation parameter $\lambda$ and the number of hidden nodes L. The main steps of Jaya-based RVFL training are listed as follows:

Step 1. Initialize population: all individuals of the Jaya algorithm are randomly generated, the ranges of the parameters are as follows: $L \in[1,1000] ; r \in[0,0.5)$.

Step 2. Run RVFL classifier with current parameters.

Step 3. Fitness evaluation: calculate the fitness value. Here, the 5-fold cross-validation accuracy classification is used as the fitness value.

Step 4. Update the parameters: if the fitness value in the current generation is better than that in the previous generation, then update the population positions and generate the next-generation populations according to the optimization mechanism of the Jaya algorithm.

Step 5. Termination criteria: if the criterion is met, go to Step 6; otherwise, increase the evolutionary generation by 1 , and then return to Step 2. Here, the criterion is that the 
TABLE 1: Input features of the dataset.

\begin{tabular}{|c|c|}
\hline No. & Input features \\
\hline Tz1 & Average mechanical power of all generators before fault \\
\hline Tz2 & Maximum value of all generators' initial rotor accelerations \\
\hline Tz3 & Initial rotor angle of the generator that has the maximum acceleration \\
\hline Tz4 & Initial average acceleration power of all generators \\
\hline Tz5 & System impact at $t_{c l}$ \\
\hline Tz6 & $\mathrm{RA}_{1}$ at $t_{c l}$ \\
\hline Tz7 & $\mathrm{KE}_{1}$ at $t_{c l}$ \\
\hline Tz8 & Rotor angle of the generator that has the maximum kinetic energy at $t_{c l}$ \\
\hline Tz9 & $\mathrm{KE}_{2}$ at $t_{c l}$ \\
\hline Tz10 & $\mathrm{KE}_{3}$ at $t_{c l}$ \\
\hline Tz11 & $\mathrm{MD}$ at $t_{c l}$ \\
\hline Tz12 & $\mathrm{RA}_{2}$ at $t_{c l}$ \\
\hline Tz13 & System impact at $t_{1}$ \\
\hline Tz14 & $\mathrm{KE}_{2}$ at $t_{1}$ \\
\hline Tz15 & $\mathrm{KE}_{3}$ at $t_{1}$ \\
\hline Tz16 & $\mathrm{RA}_{1}$ at $t_{1}$ \\
\hline Tz17 & $\mathrm{MD}$ at $t_{1}$ \\
\hline Tz18 & $\mathrm{KE}_{1}$ at $t_{1}$ \\
\hline Tz19 & $\mathrm{RA}_{2}$ at $t_{1}$ \\
\hline Tz20 & System impact at $t_{2}$ \\
\hline Tz21 & $\mathrm{KE}_{2}$ at $t_{2}$ \\
\hline Tz22 & $\mathrm{KE}_{3}$ at $t_{2}$ \\
\hline Tz23 & $\mathrm{KE}_{1}$ at $t_{2}$ \\
\hline Tz24 & $\mathrm{RA}_{1}$ at $t_{2}$ \\
\hline Tz25 & $\mathrm{MD}$ at $t_{2}$ \\
\hline Tz26 & $\mathrm{RA}_{2}$ at $t_{2}$ \\
\hline Tz27 & System impact at $t_{3}$ \\
\hline Tz28 & $\mathrm{KE}_{1}$ at $t_{3}$ \\
\hline Tz29 & $\mathrm{KE}_{2}$ at $t_{3}$ \\
\hline Tz30 & $\mathrm{KE} 3$ at $t_{3}$ \\
\hline Tz31 & $\mathrm{RA}_{1}$ at $t_{3}$ \\
\hline Tz32 & $\mathrm{MD}$ at $t_{3}$ \\
\hline Tz33 & $\mathrm{RA}_{2}$ at $t_{3}$ \\
\hline
\end{tabular}

COI: center of inertia; $t_{\mathrm{cl}}$ : the fault clearing time; $t_{1}, t_{2}$, and $t_{3}$ : the third, sixth, and ninth cycle after fault clearance; $\mathrm{RA}_{1}$ : rotor angle of the generator that has the largest difference relative to the $\mathrm{COI} ; \mathrm{KE1}$ : kinetic energy of the generator with the maximum rotor angle; $\mathrm{RA}_{2}$ : rotor angular velocity of the generator with the largest difference relative to the $\mathrm{COI} ; \mathrm{KE}_{2}$ : maximum rotor kinetic energy; $\mathrm{KE}_{3}$ : average rotor kinetic energy; $\mathrm{MD}$ : maximum difference of rotor angles. Among the features, Tz1: the load level; Tz2 $\sim$ Tz4: the features extracted at $t_{0}$; Tz5 $\sim$ Tz12: the features extracted at $t_{c}$; Tz13 Tz33: the postfault dynamic features of the system.

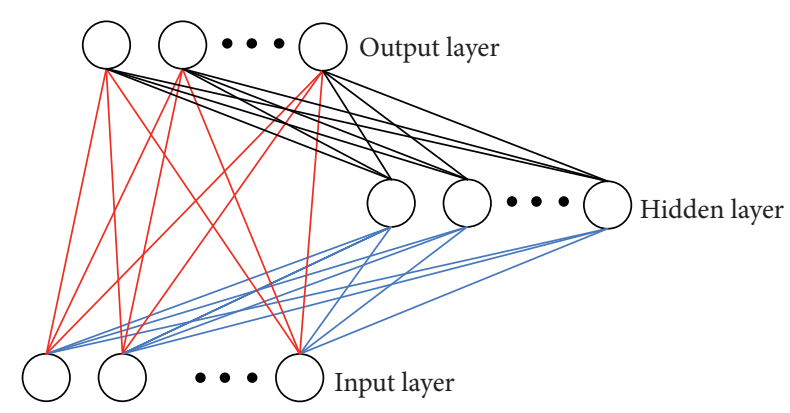

FIGURE 2: Structure of RVFL network.

current generation exceeds the maximum number of iterations or the fitness value is greater than a pregiven threshold.

Step 6. Output the optimal RVFL classifier.
3.3.2. TSA Scheme. As shown in Figure 3, the proposed scheme includes two phases: offline training and real-time assessment.

(1) Generation of the Knowledge Base. A knowledge base (KB) consists of large numbers of operating conditions (OCs), and it implies the mapping relationship between inputs and stability statuses. In this work, KB is generated by using Power System Toolbox (PST). The simulations are repeatedly performed under different OCs and faults. In each case, a label Class_Lable is defined as

$$
\text { Class_Lable }=\operatorname{sgn}\left(\frac{360^{\circ}-|\Delta \delta|_{\max }}{360^{\circ}+|\Delta \delta|_{\max }}\right),
$$

where $\operatorname{sgn}(\cdot)$ is the sign function and $\Delta \delta_{\max }$ is the maximum relative rotor angle deviation between machines after the fault occurrence. If the class label is " -1 ," the system is considered to be unstable; otherwise, the system is stable. 
(2) Feature Extraction. As illustrated in Table 1, a set of system-level features, are extracted. These features depict the transient disturbed pattern space during the whole distribution process.

(3) Data Preprocessing. The min-max normalization technique is used as the data preprocessing method, i.e.,

$$
\mathrm{val}^{\prime}=\frac{\mathrm{val}-\min _{a}}{\max _{a}-\min _{a}},
$$

where val and $\mathrm{val}^{\prime}$ are the original feature value and its scaled value and $\min _{a}$ and $\max _{a}$ are, respectively, the lower and upper bounds of the feature value.

(4) Real-Time Assessment. When new OCs are fed into the trained TSA model, the stability status will be immediately obtained. If the system is stable, then enter the next cycle; otherwise, the emergency control will be started up.

\section{Case Study}

To examine the effectiveness of our approach, the IEEE 39bus system and a real-world power system-Hebei provincial power system, China-are utilized as the testing systems. The used T-D simulation software is the Power System Toolbox (PST). All simulations are performed under the MATLAB environment on a desktop PC platform equipped with Intel Core i5-6400 2.70 GHz CPU and $8 \mathrm{~GB}$ RAM. Note that, in this study, PMU data are simulated through detailed numerical simulations via the PST.

4.1. Case 1: IEEE 39-Bus System. This system is a famous benchmark system extensively studied in the previous literature [36-44]. It includes 10 generators, 39 buses, and 46 lines, as illustrated in Figure 4.

4.1.1. KB Generation. During the simulations, three-phase short-circuit faults are created at $0.1 \mathrm{~s}$ and cleared at $0.4 \mathrm{~s}$ for all of the contingencies. After fault clearance, reclosures are successfully performed and no topology changes occur. OCs are generated by modifying the bus loads at random within $80 \%$ to $120 \%$ of the original values. The obtained total 3300 samples are divided into two subsets: wherein one that consists of 2200 randomly selected samples is for training and the other is for testing.

4.1.2. Test Results. To reasonably assess the optimization performance of the Jaya algorithm, comparison tests are carried out between the Jaya and other optimization algorithms, e.g., genetic algorithm (GA) and practice swarm optimization (PSO). At the same time, due to the inherent occasionality during the testing process, statistical tests are employed to measure the classifier performance [60]. Thus, to measure the model's classification ability, the following indicator $\eta$ is employed $[45,58]$ :

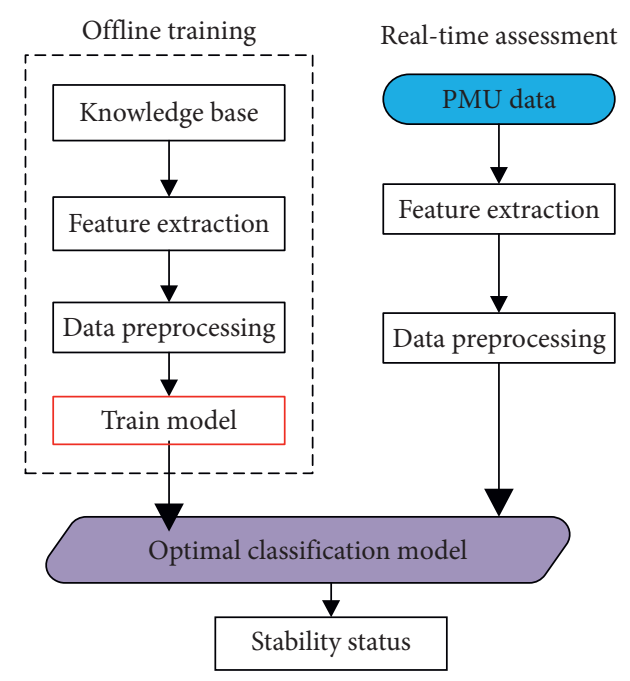

Figure 3: Proposed TSA scheme.

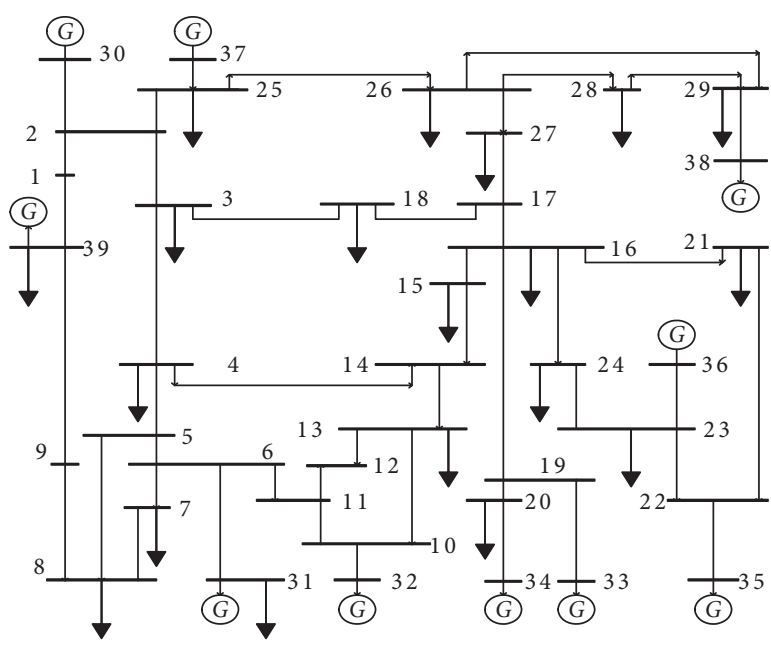

FIgURE 4: New England 39-bus system.

$$
\eta=\frac{\mathrm{Acc}+\mathrm{Kap}+\mathrm{Auc}}{3}
$$

where Acc is the test accuracy, Kap denotes the kappa statistic value, and Auc is the area under the receiver operating characteristic (ROC) curve.

The results are demonstrated in Table 2.

Table 2 shows that the presented scheme can accurately assess the stability status of the system. As a result, two conclusions can be drawn: (1) the Jaya algorithm is effective to strengthen the classification ability of the presented model, and furthermore, the Jaya algorithm is obviously superior to other alternatives such as GA and PSO.

4.1.3. Comparison with TSA Models. To further evaluate the proposed method, comparison tests with other TSA models (including MLP and SVM) are performed. The parameters in these algorithms are as follows: for MLP, the number of hidden neurons is 25 , and the back-propagation algorithm is 
TABLE 2: Test results using different optimization algorithms.

\begin{tabular}{lcccr}
\hline TSA model & Acc (\%) & Kap & Auc & \\
\hline Jaya-RVFL & 98.00 & 0.980 & 0.967 & 0.976 \\
GA-RVFL & 96.00 & 0.915 & 0.959 & 0.945 \\
PSO-RVFL & 95.45 & 0.905 & 0.955 & 0.938 \\
\hline
\end{tabular}

TABLE 3: Test results of different TSA models.

\begin{tabular}{lcccr}
\hline TSA model & Acc $(\%)$ & Kap & Auc & \\
\hline Jaya-RVFL & 98.00 & 0.980 & 0.967 & 0.976 \\
SVM & 96.18 & 0.925 & 0.964 & 0.950 \\
MLP & 95.00 & 0.895 & 0.950 & 0.932 \\
\hline
\end{tabular}

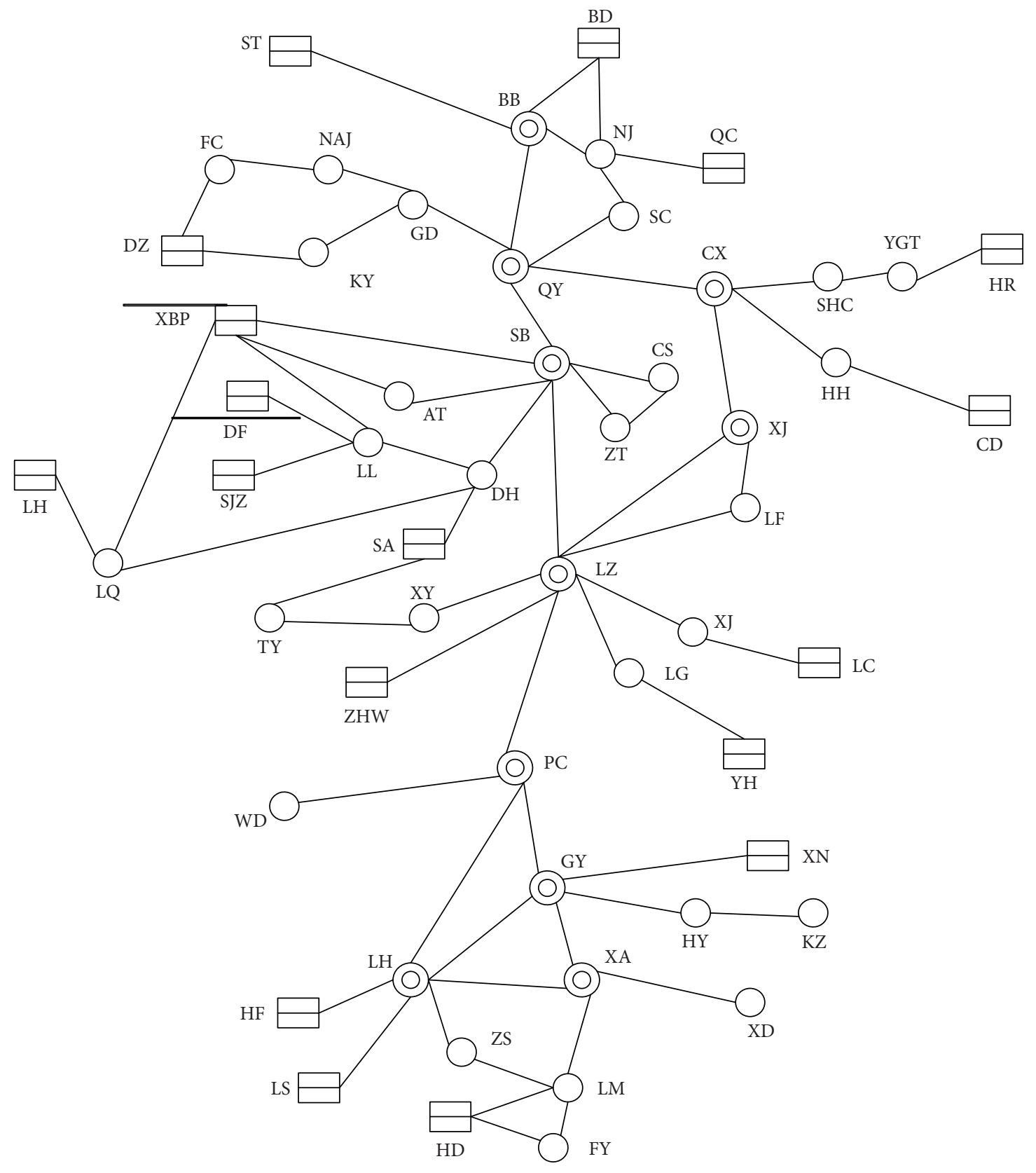

Power plant

(O) $500 \mathrm{kV}$ Power Station

$220 \mathrm{kV}$ Power Station

FIgURE 5: Hebei provincial power system. 
TABLE 4: Test results in Case 2.

\begin{tabular}{lcccc}
\hline TSA model & Acc (\%) & Kap & Auc & $\eta$ \\
\hline Jaya-RVFL & 98.09 & 0.971 & 0.960 & 0.971 \\
SVM & 96.18 & 0.921 & 0.951 & 0.945 \\
MLP & 95.29 & 0.916 & 0.948 & 0.939 \\
\hline
\end{tabular}

employed; for SVM, the used kernel function and parameter selection method are, respectively, chosen as the radial basis function kernel and the grid search algorithm [58].

The test results of different TSA models are listed in Table 3.

Table 3 indicates that the proposed approach possesses better predictive performance than all other comparative models. There are two reasons for this: (1) the RVFL network itself has good generalization ability, and (2) the performance of the RVFL-based model has been strengthened because of the parameter optimization of the Jaya algorithm.

4.2. Case 2: The Hebei Provincial Power System. In order to further evaluate the performances of our approach, an examination has been carried out on a real-world power system-Hebei provincial power system, China. This system has a total of 18 generators, 103 buses, 169 transmission lines, and 31 transformers [61]. The single-line diagram of this system is illustrated in Figure 5.

4.2.1. KB Generation. Regarding the used generators' model, 12 generators are modeled by the six-order model with the governors and the excitation systems; the rest adopts the classical third-order model. The load uses a composite model with $60 \%$ constant power and $40 \%$ constant impedance. Load levels range from $80 \%$ to $130 \%$ of the base value. The types of faults considered are threephase ground faults, which are cleared within 5 to 10 cycles after the fault occurrence. The fault locations are randomly selected on different lines. A total of 2000 samples are generated via T-D simulations; 1320 ones are selected as the training dataset, and the rest as the testing dataset.

4.2.2. Test Results. The test results with other TSA models are listed in Table 4.

Table 4 shows that the presented approach is also applicable for a practical system. Furthermore, the performance of our approach is significantly superior to that of other commonly used TSA models. It should be noted that the SVM outperforms the MLP in two cases. The reason for this phenomenon is that the SVM has better generalization ability than the MLP since it is based on the structural risk minimization principle, effectively avoiding the over-fitting problem of MLP neural networks that use the conventional empirical risk minimization principle.

\section{Conclusions}

Pattern recognition has recently proven to be effective and promising for transient stability assessment. However, many such methods suffer some inherent disadvantages such as local optima and slow convergence. In this study, a new TSA approach using RVFL network optimized by the Jaya algorithm, called Jaya-RVFL, is proposed for power systems. First of all, an RVFL-based TSA model is built to reflect the mapping relationships between the system-scale input features and the stability status. Next, the quantile scaling approach is utilized to enhance the performance of RVFL classifiers via the Jaya algorithm. The simulation results on the IEEE 39-bus system demonstrate that the proposed method manages to assess the transient stability of power system. More importantly, the classification performance of our approach outperforms that of other commonly used TSA models, such as MLP and SVM.

Our future work will focus on extending the proposed approach to estimate an accurate transient stability boundary and address stability constrains in transient stability-constrained optimal power flow. It is interesting to investigate TSA of power systems with new elements such as distributed generations $[62,63]$. Another potential topic in future research is to develop TSA model using new machine learning techniques such as deep learning [64, 65].

\section{Data Availability}

The (IEEE 39-bus system) data used to support the findings of this study are included within the article.

\section{Conflicts of Interest}

The authors declare that they have no conflicts of interest.

\section{References}

[1] P. M. Anderson and A. A. Fouad, Power System Control and Stability, Institute of Electrical and Electronics Engineers, Piscataway, NJ, USA, 2nd edition, 2003.

[2] P. Kundur, J. Paserba, V. Ajjarapu et al., "Definition and classification of power system stability," Institute of Electrical and Electronics Engineers Transactions on Power Systems, vol. 19, pp. 1387-1401, 2004.

[3] S. Yang, B. Zhang, M. Hojo, and F. Su, "An ME-SMIB based method for online transient stability assessment of a multiarea interconnected power system," Institute of Electrical and Electronics Engineers Access, vol. 6, pp. 65874-65884, 2018.

[4] Y. Li, B. Feng, G. Li, J. Qi, D. Zhao, and Y. Mu, "Optimal distributed generation planning in active distribution networks considering integration of energy storage," Applied Energy, vol. 210, pp. 1073-1081, 2018.

[5] Y. Li, Z. Yang, G. Li et al., "Optimal scheduling of isolated microgrid with an electric vehicle battery swapping station in multi-stakeholder scenarios: a bi-level programming approach via real-time pricing," Applied Energy, vol. 232, pp. 54-68, 2018.

[6] Y. Li, C. Wang, G. Li, J. Wang, D. Zhao, and C. Chen, "Improving operational flexibility of integrated energy system with uncertain renewable generations considering thermal 
inertia of buildings," Energy Conversion and Management, vol. 207, p. 112526, 2020.

[7] P. Jin, Y. Li, G. Li, Z. Chen, and X. Zhai, "Optimized hierarchical power oscillations control for distributed generation under unbalanced conditions," Applied Energy, vol. 194, pp. 343-352, 2017.

[8] Z. Qu, Y. Zhang, N. Qu, L. Wang, Y. Li, and Y. Dong, "Method for quantitative estimation of the risk propagation threshold in electric power CPS based on seepage probability," Institute of Electrical and Electronics Engineers Access, vol. 6, pp. 68813-68823, 2018.

[9] Y. Li, Y. Li, G. Li, D. Zhao, and C. Chen, "Two-stage multiobjective OPF for AC/DC grids with VSC-HVDC: incorporating decisions analysis into optimization process," Energy, vol. 147, pp. 286-296, 2018.

[10] Y. Li and S. Wu, "Controlled islanding for a hybrid AC/DC grid with VSC-HVDC using semi-supervised spectral clustering," Institute of Electrical and Electronics Engineers Access, vol. 7, pp. 10478-10490, 2019.

[11] Y. Li, Z. Yang, G. Li, D. Zhao, and W. Tian, "Optimal scheduling of an isolated microgrid with battery storage considering load and renewable generation uncertainties," Institute of Electrical and Electronics Engineers Transactions on Industrial Electronics, vol. 66, no. 2, pp. 1565-1575, 2019.

[12] Y. Li, Z. Yang, D. Zhao, H. Lei, B. Cui, and S. Li, "Incorporating energy storage and user experience in isolated microgrid dispatch using a multi-objective model," IET Renewable Power Generation, vol. 13, no. 6, pp. 973-981, 2019.

[13] Y. Li and K. Li, "Incorporating demand response of electric vehicles in scheduling of isolated microgrids with renewables using a bi-level programming approach," Institute of Electrical and Electronics Engineers Access, vol. 7, pp. 116256-116266, 2019.

[14] Y. Li, J. Wang, D. Zhao, G. Li, and C. Chen, "A two-stage approach for combined heat and power economic emission dispatch: combining multi-objective optimization with integrated decision making," Energy, vol. 162, pp. 237-254, 2018.

[15] M. Zhang and Y. Li, "Multi-objective optimal reactive power dispatch of power systems by combining classification-based Multi-objective evolutionary algorithm and integrated decision making," Institute of Electrical and Electronics Engineers Access, vol. 8, pp. 38198-38209, 2020.

[16] H. Farhangi, "The path of the smart grid," Institute of Electrical and Electronics Engineers Power and Energy Magazine, vol. 8, no. 1, pp. 18-28, 2010.

[17] V. Terzija, G. Valverde, C. Deyu Cai et al., "Wide-area monitoring, protection, and control of future electric power networks," in Proceedings of the Institute of Electrical and Electronics Engineers, vol. 99, no. 1, pp. 80-93, 2011.

[18] I. Kamwa, S. R. Samantaray, and G. Joos, "Compliance analysis of PMU algorithms and devices for wide-area stabilizing control of large power systems," Institute of Electrical and Electronics Engineers Transactions on Power Systems, vol. 28, no. 2, pp. 1766-1778, 2013.

[19] M. E. C. Bento, D. Dotta, R. Kuiava, and R. A. Ramos, “A procedure to design fault-tolerant wide-area damping controllers," Institute of Electrical and Electronics Engineers Access, vol. 6, pp. 23383-23405, 2018.

[20] J. Shi, B. Sullivan, M. Mazzola, B. Saravi, U. Adhikari, and T. Haupt, "A relaxation-based network decomposition algorithm for parallel transient stability simulation with improved convergence," Institute of Electrical and Electronics Engineers Transactions on Parallel and Distributed Systems, vol. 29, no. 3, pp. 496-511, 2018.
[21] H. D. Chiang, F. F. Wu, and P. P. Varaiya, "A BCU method for direct analysis of power system transient stability," Institute of Electrical and Electronics Engineers Transactions on Power Systems, vol. 9, pp. 1194-1208, 1994.

[22] T. L. Vu and K. Turitsyn, "Lyapunov functions family approach to transient stability assessment," Institute of Electrical and Electronics Engineers Transactions on Power Systems, vol. 31, no. 2, pp. 1269-1277, 2016.

[23] P. Bhui and N. Senroy, "Real time prediction and control of transient stability using transient energy function," Institute of Electrical and Electronics Engineers Transactions on Power Systems, vol. 32, p. 1, 2016.

[24] Y. Xue, L. Wehenkel, R. Belhomme et al., "Extended equal area criterion revisited (EHV power systems)," Institute of Electrical and Electronics Engineers Transactions on Power Systems, vol. 7, no. 3, pp. 1012-1022, 1992.

[25] Z.-B. Shi, T. Yu, Q. Zhao, Y. Li, and Y.-B. Lan, "Comparison of algorithms for an electronic nose in identifying liquors," Journal of Bionic Engineering, vol. 5, no. 3, pp. 253-257, 2008.

[26] Z.-b. Shi, Y. Li, and T. Yu, "Short-term load forecasting based on LS-SVM optimized by bacterial colony chemotaxis algorithm," in 2009 International Conference on Information and Multimedia Technology, pp. 306-309, Jeju Island, South Korea, December 2009.

[27] X. Gu and Y. Li, "Transient stability assessment of power systems based on local learning machine and bacterial colony chemotaxis algorithm," Transactions of China Electrotechnical Society, vol. 28, pp. 271-279, 2013.

[28] S. Kai, S. Likhate, V. Vittal, V. S. Kolluri, and S. Mandal, "An online dynamic security assessment scheme using phasor measurements and decision trees," Institute of Electrical and Electronics Engineers Transactions on Power Systems, vol. 22, pp. 1935-1943, 2007.

[29] M. He, J. Zhang, and V. Vittal, "Robust online dynamic security assessment using adaptive ensemble decision-tree learning," Institute of Electrical and Electronics Engineers Transactions on Power Systems, vol. 28, no. 4, pp. 4089-4098, 2013.

[30] T. Guo and J. V. Milanovic, "Probabilistic framework for assessing the accuracy of data mining tool for online prediction of transient stability," Institute of Electrical and Electronics Engineers Transactions on Power Systems, vol. 29, no. 1, pp. 377-385, 2014.

[31] D. J. Sobajie and Y. H. Pao, "Artificial neural-net based dynamic security assessment for electric power systems," Institute of Electrical and Electronics Engineers Trans. Power Syst.vol. 4, pp. 220-228, 1989.

[32] C. A. Jensen, M. A. El-Sharkawi, and R. J. Marks, "Power system security assessment using neural networks: feature selection using Fisher discrimination," Institute of Electrical and Electronics Engineers Transactions on Power Systems, vol. 16, no. 4, pp. 757-763, 2001.

[33] A. G. Bahbah and A. A. Girgis, "New method for generators' angles and angular velocities prediction for transient stability assessment of multimachine power systems using recurrent artificial neural network," Institute of Electrical and Electronics Engineers Transactions on Power Systems, vol. 19, no. 2, pp. 1015-1022, 2004.

[34] A. Karami and S. Z. Esmaili, “Transient stability assessment of power systems described with detailed models using neural networks," International Journal of Electrical Power \& Energy Systems, vol. 45, no. 1, pp. 279-292, 2013.

[35] N. Amjady and S. F. Majedi, "Transient stability prediction by a hybrid intelligent system," Institute of Electrical and 
Electronics Engineers Transactions on Power Systems, vol. 22, no. 3, pp. 1275-1283, 2007.

[36] L. S. Moulin, A. P. A. DaSilva, M. A. El-Sharkawi, and R. J. MarksII, "Support vector machines for transient stability analysis of large-scale power systems," Institute of Electrical and Electronics Engineers Transactions on Power Systems, vol. 19, no. 2, pp. 818-825, 2004.

[37] F. R. Gomez, A. D. Rajapakse, U. D. Annakkage, and I. T. Fernando, "Support vector machine-based algorithm for post-fault transient stability status prediction using synchronized measurements," Institute of Electrical and Electronics Engineers Transactions on Power Systems, vol. 26, no. 3, pp. 1474-1483, 2011.

[38] Y. Li and X. Gu, "Feature selection for transient stability assessment based on improved maximal relevance and minimal redundancy criterion," Proceedings of the CSEE, vol. 33, pp. 179-186, 2013.

[39] J. Geeganage, U. D. Annakkage, T. Weekes, and B. A. Archer, "Application of energy-based power system features for dynamic security assessment," Institute of Electrical and Electronics Engineers Transactions on Power Systems, vol. 30, no. 4, pp. 1957-1965, 2015.

[40] Y. Xu, Z. Y. Dong, K. Meng, R. Zhang, and K. P. Wong, "Realtime transient stability assessment model using extreme learning machine," IET Generation, Transmission \& Distribution, vol. 5, no. 3, pp. 314-322, 2011.

[41] Y. Zhang, T. Li, G. Na, G. Li, and Y. Li, "Optimized extreme learning machine for power system transient stability prediction using synchrophasors," Mathematical Problems in Engineering, vol. 2015, Article ID 529724, 8 pages, 2015.

[42] I. B. Sulistiawati, A. Priyadi, O. A. Qudsi, A. Soeprijanto, and N. Yorino, "Critical clearing time prediction within various loads for transient stability assessment by means of the extreme learning machine method," International Journal of Electrical Power \& Energy Systems, vol. 77, pp. 345-352, 2016.

[43] R. Zhang, K. P. Wong, Y. Xu, and Z. Y. Dong, "Post-disturbance transient stability assessment of power systems by a self-adaptive intelligent system," IET Generation, Transmission \& Distribution, vol. 9, no. 3, pp. 296-305, 2015.

[44] Y. Li and Z. Yang, "Application of EOS-ELM with binary Jayabased feature selection to real-time transient stability assessment using PMU data," Institute of Electrical and Electronics Engineers Access, vol. 5, pp. 23092-23101, 2017.

[45] Y. Li, G. Li, and Z. Wang, "Rule extraction based on extreme learning machine and an improved ant-miner algorithm for transient stability assessment," PloS one, vol. 10, no. 6, Article ID e0130814, 2015.

[46] B. Wang, B. Fang, Y. Wang, H. Liu, and Y. Liu, "Power system transient stability assessment based on big data and the core vector machine," Institute of Electrical and Electronics Engineers Transactions on Smart Grid, vol. 7, no. 5, pp. 2561-2570, 2016.

[47] Y. Li, G. Li, Z. Wang, Z. Han, and X. Bai, “A multifeature fusion approach for power system transient stability assessment using PMU data," Mathematical Problems in Engineering, vol. 201510 pages, 2015.

[48] J. Q. James, D. J. Hill, A. Y. Lam, J. Gu, and V. O. Li, "Intelligent time-adaptive transient stability assessment system," Institute of Electrical and Electronics Engineers Transactions Power Systems, vol. 33, no. 1, pp. 1049-1058, 2018.

[49] Y.-H. Pao, G.-H. Park, and D. J. Sobajic, "Learning and generalization characteristics of the random vector functional-link net," Neurocomputing, vol. 6, no. 2, pp. 163-180, 1994.
[50] L. Zhang and P. N. Suganthan, "A comprehensive evaluation of random vector functional link networks," Information Sciences, vol. 367-368, pp. 1094-1105, 2016.

[51] C. Ren, Y. Xu, Y. Zhang, and R. Zhang, "A hybrid randomized learning system for temporal-adaptive voltage stability assessment of power systems," Institute of Electrical and Electronics Engineers Transactions on Industrial Informatics, vol. 16, no. 6, pp. 3672-3684, 2020.

[52] Y. Li, Y. Li, and Y. Sun, "Online static security assessment of power systems based on lasso algorithm," Applied Sciences, vol. 8, no. 9, p. 1442, 2018.

[53] Y. Zhang, Y. Xu, and Z. Y. Dong, "Robust classification model for PMU-based on-line power system DSA with missing data," IET Generation, Transmission \& Distribution, vol. 11, pp. 4484-4491, 2017.

[54] R. V. Rao, "Jaya: A simple and new optimization algorithm for solving constrained and unconstrained optimization problems," International Journal of Industrial and Systems Engineering Computers, vol. 7, pp. 19-34, 2016.

[55] S. Mishra and P. K. Ray, "Power quality improvement using photovoltaic fed DSTATCOM based on JAYA optimization," Institute of Electrical and Electronics Engineers Transactions on Sustainable Energy, vol. 7, no. 4, pp. 1672-1680, 2016.

[56] E. E. Elattar and S. K. ElSayed, "Modified JAYA algorithm for optimal power flow incorporating renewable energy sources considering the cost, emission, power loss and voltage profile improvement," Energy, vol. 178, pp. 598-609, 2019.

[57] R. V. Rao, A. Saroj, P. Oclon, J. Taler, and J. Lakshmi, “A posteriori multiobjective self-adaptive multipopulation Jaya algorithm for optimization of thermal devices and cycles," Institute of Electrical and Electronics Engineers Access, vol. 7, pp. 4113-4134, 2019.

[58] X. Gu, Y. Li, and J. Jia, "Feature selection for transient stability assessment based on kernelized fuzzy rough sets and memetic algorithm," International Journal of Electrical Power \& Energy Systems, vol. 64, pp. 664-670, 2015.

[59] Y. Li, G. Li, X. Gu, and Y. Zhang, "Transient stability assessment of power systems based on ensemble OS-ELM," Transactions of China Electrotechnical Society, vol. 30, no. 14, pp. 412-418, 2015.

[60] D. N. Reshef, Y. A. Reshef, H. K. Finucane et al., "Detecting novel associations in large data sets," Science, vol. 334, no. 6062, pp. 1518-1524, 2011.

[61] S. Grossman, X. Gu, G. Zhou, and Y. Li, "Optimisation and comprehensive evaluation of alternative energising paths for power system restoration," IET Generation, Transmission \& Distribution, vol. 13, no. 10, pp. 1923-1932, 2019.

[62] I. Xyngi, A. Ishchenko, M. Popov, and L. Van Der Sluis, "Transient stability analysis of a distribution network with distributed generators," Institute of Electrical and Electronics Engineers Transactions on Power Systems, vol. 24, no. 2, pp. 1102-1104, 2009.

[63] M. A. Chowdhury, N. Hosseinzadeh, H. R. Pota, and W. Shen, "Transient stability of power system integrated with doubly fed induction generator wind farms," IET Renewable Power Generation, vol. 9, no. 2, pp. 184-194, 2015.

[64] Z. Tang and Z. Zhang, "The multi-objective optimization of combustion system operations based on deep data-driven models," Energy, vol. 182, pp. 37-47, 2019.

[65] L. Zhu, D. J. Hill, and C. Lu, "Hierarchical deep learning machine for power system online transient stability prediction," Institute of Electrical and Electronics Engineers Transactions on Power Systems, vol. 35, no. 3, pp. 2399-2411, 2020. 